\title{
Intertextualidad en los márgenes: las formas de la cita en las glosas de la Crónica de la población de
} Ávila

\section{Manuel Abeledo}

\section{OpenEdition}

\section{Journals}

Edición electrónica

URL: http://journals.openedition.org/agedor/457

DOI: $10.4000 /$ agedor.457

ISSN: 2104-3353

\section{Editor}

Laboratoire LISAA

Referencia electrónica

Manuel Abeledo, « Intertextualidad en los márgenes: las formas de la cita en las glosas de la Crónica de la población de Ávila », L'Ấge d'or [En línea], 8 | 2015, Publicado el 01 febrero 2016, consultado el 05 mayo 2019. URL : http://journals.openedition.org/agedor/457 ; DOI : 10.4000/agedor.457 


\title{
Intertextualidad en los márgenes: las formas de la cita en las glosas de la Crónica de la población de Ávila
}

\begin{abstract}
Resumen: Ce travail analyse les trois gloses d'un lecteur de ca. 1700 dans le manuscrit 11/8544 de la Real Academia de la Historia, qui contient le texte de la Crónica de la población de Avila. Les trois gloses sont des citations presque textuelles d'un manuscrit de Bartolomé Fernández Valencia de 1676. Cette filiation nous permet, en plus d'éclairer la circulation des textes historiographiques à propos d'Ávila à cette époque, de résoudre quelques errata dans notre édition du texte et dans un article écrit auparavant à propos des gloses de ce manuscrit, de nous interroger à propos de la nature de la citation textuelle dans l'espace marginal, et sur les formes d'appropriation d'un texte de la part des glossateurs.
\end{abstract}

Palabras clave : Historiographie médiévale - Ávila - Histoire de Castille - Glose - Marginalia - Critique textuelle - Transcription

Resumen: El presente trabajo analiza las tres glosas dejadas por una mano de ca. 1700 en el manuscrito 11/8544 de la Real Academia de la Historia, conteniendo el texto de la Crónica de la población de Ávila. Las tres glosas son citas más o menos textuales de un manuscrito de Bartolomé Fernández Valencia de 1676. Tal filiación permite, además de iluminar la circulación de los textos historiográficos sobre Ávila en esos años, y de resolver algunas erratas a mi edición del texto y a un artículo que escribí anteriormente sobre las glosas de este manuscrito, hacerse algunas preguntas acerca de la naturaleza de la cita textual en el espacio marginal, y los modos de apropiación de un texto por parte de los glosadores.

Palabras clave: Historiografía medieval - Ávila - Historia de Castilla - Glosa - Marginalia - Crítica textual - Transcripción

Publiqué en el año 2010, en el número 30 de la revista Incipit, un artículo ${ }^{1}$ en el que analizaba las glosas presentes en el manuscrito C (11/8544 de la Real Academia de la Historia) de la Crónica de la población de Ávila, en el marco de mi edición del texto que vio la luz, finalmente, en $2012^{2}$. La intención principal de ese artículo fue la de argumentar el enorme interés que tienen las glosas, la importancia de su estudio y la necesidad de su inclusión en las ediciones críticas de textos medievales; citaba allí un pasaje de David Hook que vale la pena repetir aquí:

If a book, printed or manuscript, contains later annotations to the text, this is an essential dimension not only of the history of the individual volume as a physical object, but of the intellectual history of the text or texts contained therein, and of the society in which the annotator lived ${ }^{3}$.

\footnotetext{
1 ABELEDO, Manuel, «Sobre el valor de los marginalia en la tradición manuscrita. Análisis del ms. 11/8544 de la Real Academia de la Historia de la Crónica de la población de Ávila», in Incipit, n³0, Buenos Aires, SECRIT, 2010, p. 111-132.

2 ABELEDO, Manuel (ed.), Crónica de la población de Ávila, Buenos Aires, SECRIT, 2012.

${ }^{3}$ HOOK, David, «Method in the Margins: An Archeology of Annotation», in A. Beresford y A. Deyermond (eds.), Proceedings of the Eighth Colloquium, Londres, Department of Hispanic Studies, Queen Mary and Westfield College, 1997, p. 135-144, cita en p. 135.
} 
Y quise, en particular, atribuir ese interés a todos los marginalia existentes, sin tener que limitarse a considerar las pocas inscripciones que efectivamente transmiten información de interés. Quise ejemplificar este punto con una abundantísima serie de glosas que constituyen una transcripción al margen de varias manos del texto de la Crónica casi en su completitud en el manuscrito antedicho. Es difícil pensar que una intervención pueda aportar algo menos interesante que una copia literal del texto, y sin embargo, era mi intención evidenciar que muchos elementos de interés pueden deducirse de ello. Son tres manos las que efectúan esta transcripción, llamadas en su momento, según su orden de aparición, G1, G2 y G3. Su orden cronológico es exactamente el inverso, como argumenté en su momento basándome en el modo en que se acomodan sus intervenciones en el espacio del margen. Estas transcripciones, en aquel artículo, me permitieron desarrollar algunas cuestiones de interés en torno al funcionamiento de la actividad glosadora y de mecánicas de la lectura. De su estudio se desprendía la posibilidad de pensar los modos de recepción del texto, y en términos más generales se deducía, por ejemplo, que la práctica de la transcripción estaba fuertemente condicionada tanto por cuestiones mecánicas en la conformación del códice y de su copia como por la actividad de los glosadores anteriores, mostrando cómo la transmisión manuscrita del texto era determinante para sus modos de lectura, poniendo en evidencia las limitaciones (inevitables, lamentablemente) de la edición impresa, en línea con cuestiones que argumenta, por ejemplo, John Dagenais ${ }^{4}$. Dado que ninguno de los glosadores era medieval, y del último de ellos se podía demostrar que había intervenido a principios del siglo XIX (y que se trata de un texto recién puesto en letras de molde por vez primera en $1913^{5}$ ), también estos fenómenos ponían en evidencia la importancia que esos modos de la transmisión manuscrita tenían aún en tiempos relativamente recientes, muchos siglos pasados ya desde los inicios de la Galaxia Gutenberg.

La intención del presente trabajo es en buena medida la de continuar ese análisis. Quisiera centrarme, en particular, en la labor del primero de esos transcriptores, el que denominé G3, pero en este caso ya en sus glosas de contenido más que en su copia marginal del texto. Una de ellas, la que aparece en folio $28 \mathrm{v}^{6}$ y cuenta un episodio ocurrido en torno a la iglesia de San Leonardo, termina con una referencia textual explícita, que en su momento transcribí así: «Vid Valencia, N. S. epitom. brand. Ab. Herm. S. Segundo ${ }^{7} . »$ La dificultad de lectura y la falta de ingenio no me permitieron en su momento deducir la referencia. Pero más tarde, considerando la posibilidad de que no fuera «N. S.» sino «M. S.», que la abreviatura fuera «grand.» en vez de «brand.» y que «Ab.» no fuera la preposición latina sino abreviatura de «Ávila» logré encontrar el texto que mentaba: el pasaje referido a la Ermita de San Segundo del Epitome de las grandezas de Ávila de Bartolomé Fernández Valencia, texto manuscrito compuesto en 1676 y editado por vez primera en tiempos recientes ${ }^{8}$.

\footnotetext{
${ }^{4}$ DAGENAIS, John, The Ethics of Reading in Manuscript Culture: Glossing the 'Libro de buen amor", Princeton, University Press, 1994.

${ }^{5}$ FORONDA Y AGUILERA, Manuel (ed.), "Crónica inédita de Ávila », in Boletín de la Real Academia de la Historia, 63 (1913), p. 110-143. Se trata, precisamente, de una transcripción del manuscrito que trabajamos aquí (y que no fue el usado como manuscrito base para ninguna de las ediciones posteriores, tampoco la mía).

${ }^{6}$ Para el texto de la Crónica utilizo aquí la misma foliación que seguí en el artículo y la edición ya mencionados: comienza en el título en fol. 1r y sigue en el índice del volumen que ocupa los fols. 2r-7r. En 9r comienza la introducción (aquí se inicia la foliación propia del volumen, por lo que es ocho números inferior a la mía hasta que se altera por ciertas anomalías) y en $10 \mathrm{r}$ comienza el texto que nos interesa aquí, que termina en $83 \mathrm{v}$ al final del apéndice «De la lealtad de los caualleros de Avila.»

7 ABELEDO, Manuel, Crónica, op. cit., p. 33, en nota.

${ }^{8}$ SOBRINO CHOMÓN, Tomás (ed.), Bartolomé Fernández Valencia, Historia y grandezas del insigne templo, fundación milagrosa, basílica sagrada y célebre santuario de los santos mártires hermanos San Vicente, Santa Sabina y Santa Cristeta: consagrado a la eterna memoria de sus inclitos nombres en el mismo lugar en que ofrecieron por Cristo sus vidas y adonde en majestuosos sepulcros son venerados sus santos cuerpos y preciosas reliquias. Contiene la invención maravillosa de la apostólica imagen de la Virgen nuestra Señora con título de la Soterraña, aparecida en el mismo templo; y por indicios y conjeturas se infiere que fue traida por los apóstoles y pintada
} 
El descubrimiento del texto de Fernández Valencia me permitió, en primer lugar, descubrir que en mi artículo de 2010 había cometido algunos errores, y aprovecho este espacio como lugar para la fe de erratas. En primer lugar, desde ya, debiera haber sumado en su momento el texto de Fernández Valencia a los de Ariz y Ayora, que consideré como posibles influencias en la lectura de los glosadores ${ }^{9}$. En segundo lugar, la fecha de 1676 da una nueva fecha ante quem para las glosas de G3, cuya datación hasta aquí se había basado exclusivamente en el estudio de la caligrafía, llegando a una conclusión evidentemente imposible: es claro que no se trata de la primera mitad del siglo XVII, como exponía alli1 ${ }^{10}$, sino que los marginalia son, al menos (y probablemente), del último cuarto del siglo. Dado que las glosas de G2 son claramente posteriores, esto también nos lleva a precisar la datación allí planteada ${ }^{11}$ (segunda mitad del s. XVII) y a pensar que también es posterior a 1676, y probablemente algo más tardío que lo planteado en su momento, si no se quiere forzar las fechas. Además, atribuí en su momento ${ }^{12}$ la glosa que aparecía en f. 15r a G3 por similitudes de letra, aunque tal similitud resultaba dudosa. Esas dudas, sumadas al hecho de que el glosador cita información de otra crónica que no es la de Fernández Valencia, me inclinan ahora a atribuirla a una mano diferente. Por otra parte, al comprobar que las tres glosas que sigo considerando de G3 provienen todas directamente del texto de Fernández Valencia, nos permite corregir algunas lecturas. La deducción según la cual G3 muestra en sus conocimientos ser habitante de Ávila ${ }^{13}$ se revela falsa, ya que no los extrae de su hábitat sino del manuscrito de Fernández Valencia (aunque el mismo conocimiento de este manuscrito, fuertemente ligado a Ávila, podría llevarnos a la misma conclusión). Si bien la fecha que aparece en la primera de ellas, en f. 26r, pareciera ser efectivamente la de 1171, como transcribí en mi edición ${ }^{14}$, en el texto de Fernández Valencia figura como $1174^{15}$. En esa misma glosa, el cambio de letra al pasar a lengua latina, cotejada con otros pasajes del códice, me había hecho suponer que la referencia era completada por $\mathrm{G}^{16}{ }^{16}$; al ver ahora que se trata en su conjunto de una transcripción literal de Fernández Valencia, semejante palimpsesto resulta mucho más improbable. Por último, en la tercera, para la glosa que figura en f. $31 \mathrm{v}$, la abreviatura $\left\langle\mathrm{m}^{\circ}\right.$. Dia», que interpreté en su momento (suponiendo alguna clase de errata) como «monasterio Dia» ${ }^{17}$, la lección correcta se revela en el texto de Fernández Valencia como «Mediodía».

Hasta aquí las erratas. Presento a continuación las tres glosas, cotejadas con sus pasajes correspondientes del texto de Fernández Valencia. En las primeras indico entre paréntesis folio y número de página de mi edición, en los segundos el número de página de la edición de Sobrino Chomón.

\begin{tabular}{|l|l|l|}
\hline 1 & El epitafio que se halló en el sepulcro de Sancho & En ella [en la Parroquia de Santiago] están \\
Ximeno en la Parroquia de Santiago de Ávila & enterrados los esforzados adalides Gómez \\
dice: Hic iacet Santius Jimenez, gemma omnium & Ximeno y Sancho Ximeno, iguales en el \\
Hispanie dux et famossus miles, qui XXVI & valor y en la sangre, hallándose el uno en \\
vicibus dux eorum extitit, qui inter sarracenos & diez y ocho lides y el otro en veinte y seis \\
obiit anno Domini 1171. & batallas, como se reconoció en el epitafio \\
El epitafio del sepulcro de Gómez Ximeno que & siguiente que se halló en su sepulcro:
\end{tabular}

por San Lucas. Con un comentario o epílogo de las grandezas de esta ciudad, Ávila, Institución Gran Duque de Alba y Caja de Ahorros de Ávila, 1992.

${ }^{9}$ ABELEDO, Manuel, «Sobre el valor...», op. cit., p. 118, 125 y 127.

${ }^{10}$ Ibidem, p. 114.

${ }^{11}$ Ibidem, p. 117.

12 ABELEDO, Manuel, Crónica, op. cit., p. 14, en nota. Vid. SOBRINO CHOMÓN, Tomás, Historia y Grandezas, op. cit., p. 31 .

${ }_{13}$ ABELEDO, Manuel, Crónica, op. cit. , p. 116.

${ }^{14}$ Ibidem, p. 29, en nota.

${ }^{15}$ SOBRINO CHOMÓN, Tomás, Historia y Grandezas, op. cit., p. 51.

${ }^{16}$ ABELEDO, Manuel, Crónica, op. cit, p. 29, en nota.

${ }^{17}$ Ibidem., p. 36, en nota. 


\begin{tabular}{|c|c|c|}
\hline & $\begin{array}{l}\text { se halló en dicha parroquia de San Santiago está } \\
\text { en versos latinos, en que se recuentan sus } \\
\text { hazañas, y por ser muy largo no se pone aquí. } \\
\text { (f. 26r-v, p. 29) }\end{array}$ & $\begin{array}{l}\text { Hic iacet Sanctius Jimenez Gemma omnium } \\
\text { Hispaniae Dux et famosus miles qui XXVI } \\
\text { vicibus dux eorum extitit qui inter sarracenos } \\
\text { obiit Anno Domini } 1174 \text {. } \\
\text { El de Gómez Ximeno está en versos latinos } \\
\text { en que se recuentan sus hazañas y victorias } \\
\text { con elegante estilo y erudición; y por ser muy } \\
\text { largo no lo pongo en este lugar. (p. } 50-51 \text { ) }\end{array}$ \\
\hline 2 & $\begin{array}{l}\text { Era la iglesia de san Leonardo media legua (más) } \\
\text { de Ávila, donde iban en procesión dos cofradías, } \\
\text { la de san Sebastián, sita en la iglesia de san } \\
\text { Segundo, y la de la Soterraña de la parroquia de } \\
\text { san Silvestre, ahora combento de carmelitos } \\
\text { calzados. Cuéntase por caso maravilloso de que } \\
\text { viniendo estas dos cofradías en procesión desde } \\
\text { la iglesia de san Leonardo, estando para asalta[r] } \\
\text { a Âvila un fiero escuadrón de moros, estos } \\
\text { viendo venir la procesión juzgaron era algún } \\
\text { poderoso exército, con que temerosos trataron } \\
\text { de retirarse y desistir del intento. Vid Valencia, } \\
\text { Ms. Epítom. Grand. Ab. Herm. S. Segundo. } \\
\text { (f. } 28 v-29 v, \text { p. 33) }\end{array}$ & $\begin{array}{l}\text { Viniendo esta hermandad la de San } \\
\text { Sebastián] y la de la Soterraña de la parroquia } \\
\text { de San Silvestre en procesión desde la iglesia } \\
\text { de san Leonardo, media legua de la ciudad, } \\
\text { estando para asaltarla un escuadrón de } \\
\text { moros, viendo venir la procesión juzgaron } \\
\text { era algún poderoso ejército; con que } \\
\text { temerosos trataron de retirarse y desistir del } \\
\text { intento. (p. 91) }\end{array}$ \\
\hline 3 & $\begin{array}{l}\text { En la parroquia de Santiago de Ávila se halló el } \\
\text { antiguo sepulcro del celebrado Nalvillos } \\
\text { Blásquez en la pared de la puerta del Mediodía, } \\
\text { y la piedra de su inscripción llevó por cosa de } \\
\text { notable antigüedad don Pedro D'Ávila, primer } \\
\text { marqués (mayordomo de Carlos V y de Felipe } \\
\text { II) de las Navas, según lo afirma el doctor } \\
\text { Alcázar en su Mussa Avilesa. (f. } 31 \mathrm{v}-32 \text { r, p. } 36 \text { ) }\end{array}$ & $\begin{array}{l}\text { En ella se halló el antiguo sepulcro del } \\
\text { celebrado Nalvillos Blázquez, en la pared de } \\
\text { la puerta del Mediodía, y la piedra de su } \\
\text { inscripción llevó por cosa de notable } \\
\text { antigüedad don Pedro Dávila, primer } \\
\text { marqués de Las Navas, según lo afirma el } \\
\text { doctor Alcázar en su Musa Avilesa. (p. 50) }\end{array}$ \\
\hline
\end{tabular}

Como se ve, la base de las tres glosas es una cita textual de Fernández Valencia, pero en todos los casos con algunas variantes. En el caso de la primera de ellas, a pesar de que G3 encuentra en Fernández Valencia ambos textos de corrido, coloca el segundo párrafo, referido a Gómez Ximeno, más abajo en la plana, a continuación de una glosa preexistente, aún si le quedaba algo de espacio a continuación del primero. Evidentemente, prefirió que la mención de los epitafios quedara cada una en un solo bloque, antes de privilegiar la continuidad de la cita. De esta manera, el glosador introduce modificaciones que tienen que ver con recuperar referencias, evitando las alusiones anafóricas explícitas o implícitas en el texto de Fernández Valencia. Otra variante que introduce en este primer caso es la omisión de «y victorias con elegante estilo y erudición», en una muy probable voluntad de síntesis. Por otra parte, hay una clara voluntad de reducir la referencia inicial que da marco a los epitafios en el texto de Fernández Valencia. Esto no obedece solamente a problemas de extensión, sino a evitar redundancias, ya que mucho de lo que dice ahí Fernández Valencia está en el texto de la crónica, que dice:

E Sancho Ximeno e Gómez Ximeno los adalides yazen soterrados en la iglesia de Santiago, e está escripto en unas piedras sobre ellos de las faziendas en que se acertaron con los cavalleros de Ávila, e Sancho Ximeno acertosse en diez y ocho lides campales, e Gómez Ximeno con él, e después que murió Sancho Ximeno viscó gran tiempo después Gómez Ximeno e cumplió sobre estas lides fasta veinte y cinco lides ${ }^{18}$.

\footnotetext{
${ }^{18}$ Ibidem, p. 29.
} 
La voluntad de G3 no es sorprendente: Fernández Valencia introduce los epitafios mediante una serie de informaciones que en la crónica ya están presentes, y no es necesaria repetirlas, por la que realiza su propia intervención para presentar, de la manera más escueta posible, el epitafio a continuación. No es sorprendente salvo que se repare en el hecho de que la información que encuentra en Fernández Valencia y la que está en la crónica son contradictorias. Es necesario repasar los datos. Según la Crónica, Sancho Ximeno combatió en dieciocho batallas, y Gómez Ximeno combatió en todas éstas y en veinticinco más, es decir, un total de cuarenta y tres. Según el padre Luis Ariz, en su texto historiográfico de 1607, que muestra haber manejado nuestra Crónica como fuente en varios pasajes, y que es posible que haya sido la fuente de Fernández Valencia, «no contentos los dos adalides de Ávila, aviéndose hallado en veynte y cinco batallas, entraron el año de mil y ciento y sesenta y quatro por tierra de Moros, corriendo la tierra de la Serepa ${ }^{19}$ ", de manera que, sumando esta última, habrían peleado ambos en veintiséis batallas. Dice el padre Ariz que esta información la extrae de la historia de África de Mármol, es decir, de la Descripción general de Africa de Luis del Mármol Carvajal, cuyo primer volumen publica en 1573. Allí dice Mármol:

Despues desto, Sancho Ximenez, y su hermano, en el año de mil y ciento y sessenta y quatro, entraron en tierra de moros, y corriendo la tierra de la Serena, sacaron della gran presa de ganados, y viniendo contra ellos los Moros de aquella comarca uvieron batalla, y los vencieron, y cargados de despojos se bolvieron a la ciudad de Avila ${ }^{20}$.

Poco antes en la misma página Mármol relata hazañas de los hermanos ocurridas en 1160, pero en ningún lugar habla de una cantidad de batallas, así que esto corre por cuenta de Ariz, que probablemente haya entendido que ésta era la última, y que por eso, dada la información del epitafio, debería haber veinticinco anteriores. No reconoce tampoco Ariz diferencia entre ambos hermanos en el número de batallas peleadas, ya que sostiene que ambos murieron en el mismo año: «parece que murió peleando con los moros, en el mismo año de 1174 que su hermano Gomez Ximeno por un epitaphio de su sepultura ${ }^{21} »$, epitafio que es el mismo que tenemos transcripto en la glosa.

Ahora bien, Fernández Valencia sostiene, como vimos, que uno participó en dieciocho batallas y el otro en veintiséis, y dada la cita del epitafio, parece ser que el segundo es Sancho Ximeno. No logro encontrar la fuente de la que Fernández Valencia ha llegado a esta información. Sólo tengo registro de los epitafios en el texto del padre Ariz, del que se está apartando en este caso. Pero sea cual sea la información que da, es posible sospechar que la presencia del número dieciocho implica alguna interferencia de la Crónica en la tradición, que es el único testimonio que presenta esa cifra, aunque aplicada a Sancho Ximeno, y no a su hermano. En cualquier caso, ninguna de estas cuentas aparece en la glosa que queremos analizar, sino que en ella sólo se dice, en el epitafio citado, que Sancho Ximeno se acertó en veintiséis lides. Puesto en orden: G3 está leyendo un texto que dice que Sancho peleó en dieciocho ocasiones, y su hermano en cuarenta y tres. Lo está cotejando con otro que dice Sancho combatió en veintiséis ocasiones y Gómez en dieciocho. Y decide copiar de allí, sin aclaración, un epitafio que dice solamente que Sancho peleó veintiséis lides. La pregunta fundamental parece ser: ¿a quién le cree G3? Si se inclinara por nuestra Crónica, probablemente tendría alguna duda en la referencia citada, que lo llevaría a no darle veracidad al epitafio y ahorrarse la transcripción, o al menos a agregar algún cuestionamiento. Si confiara, en cambio, en Fernández Valencia, sería una excelente

\footnotetext{
19 ARIZ, Luis, Historia de las grandezas de la ciudad de Ávila, Ávila, Caja General de Ahorros y Monte de Piedad de Ávila, 1978, p. 329.

${ }^{20}$ DEL MARMOL CARVAJAL, Luis, Primera parte de la descripción general de Affrica: con todos los successos de guerras que a auido entre los infieles y el pueblo christiano y entre ellos mesmos desde que Mahoma inventó su secta hasta el año del señor mil y quinientos y setenta y uno, Granada, René Rabut, 1573, p. 175.

${ }^{21}$ ARIZ, Luis, Historia de las grandezas, op. cit., p. 330.
} 
oportunidad para corregir al margen las cifras de la Crónica, en especial las de Gómez Ximeno, que no se resuelven con la glosa. Sin embargo, no hace ninguna de ambas cosas. Deja al margen información contradictoria, y omite al margen información contradictoria.

Dejemos esto al margen por un momento, y veamos el elemento que probablemente más llame la atención en la glosa, y que no es aquello que modifica u omite sino lo que copia casi textualmente: el «aquí». ¿Qué quiere decir «aquí» en la glosa de G3? Fernández Valencia dice no copiar el epitafio de Gómez Ximeno «por ser muy largo.» No hay razones para no creerle: el texto de Ariz, que puede haber sido su fuente, como ya dijimos, transmite ambos epitafios. El de Sancho Ximeno aparece exactamente igual que en la glosa y en Fernández Valencia, y el de Gómez Ximeno cuenta con catorce líneas y la fecha, mientras el de su hermano no contaba con más de tres versos y la fecha ${ }^{22}$. Es visiblemente más extenso, en efecto, y sin embargo, es evidente, no es ésa la razón por la que no lo incluye G3, sino que sencillamente no lo hace porque no está en su fuente. La referencia está cubierta así de una deixis confusa: el «aquí» remplaza, con una variación formal sin implicancias semánticas el «en este lugar» de Fernández Valencia, y el impersonal «no se pone» elude la primera persona del «no lo pongo.» No me parece aventurado suponer que estas paráfrasis, que pocas implicancias tienen en significado, estilo o extensión en un pasaje que viene citando con celosa literalidad, dan cuenta de un cierto pudor a la hora de la primera persona, a la hora de mentir haciendo propias las causas ajenas. Sin embargo, y esto es lo llamativo, tal pudor no evita la mentira, ni la matiza siquiera: sigue explicitando una razón de ser a la ausencia que no es la real. Parece ser un caso particularmente intrincado del problema de autoría de la glosa que trabaja Anne Grondeux:

La glose constitue en effet un genre littéraire où la notion d'auteur fonctionne particulièrement mal. Elle est le secteur par excellence de la création continue à plusieurs mains très souvent anonymes, et c'est en retour la notion même d'«auteur» qui fait des gloses un monde difficile et souvent décourageant ${ }^{23}$.

No supongo por esto que G3 tenga la voluntad de mentir, que quiera poner excusas a su ignorancia, que esté tratando de plasmar un engaño. Todo por el contrario. Creo que el pasaje lo obliga a hacer algo mucho más grave, que es poner en cuestión el problema de la literalidad. ¿Qué significa copiar algo literalmente para G3? Nosotros no tendríamos este problema. Un par de comillas, una referencia bibliográfica más o menos detallada es suficiente para señalarlo. Tampoco tendría este problema ningún glosador (ni copista) medieval: si lo que dice el texto es una verdad merece su lugar en el margen, sin importar primeras personas ni contingencias, y de su letra se le cambiará lo que haga falta para que eso sea lo único que tiene que ser: una verdad, sin obstáculos ni matices, cuya literalidad o falta de ella no reviste ningún valor. Pero G3 está pensando las cosas, evidentemente, de manera distinta. Conoce la referencia a la fuente, evidentemente, porque la usa con notoria pulcritud en su segunda glosa. Curiosamente, en aquella cuya transcripción es menos fiel.

El caso de la segunda glosa también es complejo. En primer lugar, tiene un gesto similar para hacer del texto presentado uno autónomo, reorganizando la sintaxis. Pero añade, a su vez, información ( $\mathrm{y}$ estas dos son las modificaciones sustanciales que efectúa en la tercera glosa), extraída esta vez sí probablemente de su acervo (la residencia de la cofradía de san Sebastián, la situación actual de la parroquia de san Silvestre). Corrige incluso, cuando precisa la distancia de Ávila de la iglesia de san Leonardo. Pero al mismo tiempo que tiene estas pretensiones de severidad sobre la información, conduce el relato hacia el terreno de las formas ficcionales y

\footnotetext{
22 Ibidem, p. 330.

${ }^{23}$ GRONDEUX, Anne, "Auctoritas et glose: quelle place pour un auteur dans une glose?», in M. Zimmerman, Auctor et auctoritas: Invention et conformisme dans l'écriture médiévale. Actes du colloque de Saint-Quentin-en-Yvelines (14-16 juin 1999), Paris, École des Chartes, 2001, p. 245-254, cita en p. 246.
} 
legendarias; podría decirse que el agregado de la introducción «cuéntase por caso maravilloso»es, precisamente, una puesta en duda del relato que se está contando y de la veracidad de su historia, en términos más bien despectivos. Pero es difícil pensar que G3 se tomara el trabajo de copiar una referencia de otro texto (cosa que no hace ni sistemática ni frecuentemente, ni mucho menos) sólo para ponerla en duda a continuación. Además, en el mismo sentido aplica el calificativo «fiero» al escuadrón de moros, lo que difícilmente tenga otra intención que la de añadir emotividad al carácter extraordinario de lo relatado. Llama entonces particularmente la atención la última de las modificaciones operadas sobre esta segunda glosa, que es la referencia explícita y detallada de la fuente del relato, único caso en toda la crónica incluyendo al resto de los glosadores. La precisión de la referencia, así como la formalidad de su tono, indican, me parece, de manera evidente, un particular interés en la fuente, precisamente en la transcripción, de las tres, que más varía y que menos carácter de veracidad histórica le da al elemento que relata. La forma de la referencia revela, a su vez, que muy probablemente la transcripción se haya hecho no sólo como recordatorio para el mismo glosador, sino también como aporte informativo para futuros lectores del códice. Digamos, entonces, que la referencia formal, que para nosotros indica precisamente objetividad de la información y reproducción literal de la palabra ajena, aparece aquí en una glosa que, al menos comparada con las otras dos, llama la atención precisamente por lo contrario: por ser la que más interviene en el texto, la que más involucra el conocimiento, la experiencia y la subjetividad de la primera persona del narrador, y al mismo tiempo la que menos grado de objetividad y veracidad histórica pretende dar a los sucesos que refiere.

El fenómeno de ese «aquí» de la primera glosa era claramente el de una supresión de los valores deícticos de los términos, y suprimir la deixis equivale a suprimir la coordenada de tiempo y espacio en el que se produce. La revelación de Fernández Valencia no es válida en referencia a su texto, sino en un valor de verdad general. Las razones que aduce no son válidas según su voluntad y circunstancia, sino válidas en sí mismas. Si el epitafio de Gómez Ximeno es demasiado extenso para incluirse en el texto de Fernández Valencia, es demasiado extenso en términos absolutos. Llama la atención, en efecto, la coincidencia de preocupaciones en ese sentido: un texto que fuera demasiado nutrido para la fuente sería con toda seguridad, a su vez, excesivo para el espacio marginal de la glosa, y ahí se ve que, efectivamente, esa afirmación es la de una verdad trasladable.

Pero al mismo tiempo la ficcionalización del relato de la segunda glosa muestra que no sólo el «aquí» de la primera entra en cuestión, sino el significado mismo de su valor de verdad. Ese texto original, ese enunciado de Fernández Valencia no es verdadero en función de su veracidad histórica, sino verdadero en su puro valor textual, es verdadero en tanto es inobjetablemente verdadero que eso ha sido escrito y, por ende, es verdadero que su enunciado pertenece al ámbito de lo pertinente al pensar en las crónicas de Ávila. El margen se transforma entonces para G3 en el ámbito de una verdad textual o, mejor dicho, intertextual, en un espacio de la verdad discursiva, en donde el objeto es en cualquier caso el universo de lo dicho. Poco importa si los epitafios efectivamente existen o existieron, si el de Nalvillos está en la pared Sur del monasterio, si dos cofradías hicieron retroceder un ataque de moros: esa información merece estar en la glosa porque ha sido dicha. Este último caso parece ser el más revelador: G3 efectivamente no parece estar buscando la historia en esa transcripción, sino que dota al relato de los atributos de la leyenda, de lo legendum, de lo que ha sido y debe ser leído. Esto permite al mismo tiempo responder al problema que traía la cifra de batallas en el epitafio de Sancho Ximeno: no está ahí para aportar datos, ni para hacer cuentas que poco importan. Está ahí para hacer presente un texto que existe, que ha sido escrito y, si se cree en su fuente, que no es ni más ni menos que la inscripción en piedra de un monumento mortuorio. Su valor es un valor, si se quiere, fetichista: es un objeto textual preciado por muchas otras cosas más que por su contenido. Lo cual permite retomar lo que veíamos antes en relación con la complejidad de la noción de autor que recubre a estas glosas. Como dice Jesús D. Rodríguez Velasco: 
La función autor no recubre al glosador, el cual es, por definición propia, un lector, un pedagogo que contribuye a la comprensión de las ideas de otro, que, ese sí, es un autor. Pero si el glosador no busca construir su idea de autor, o reconoce, al quedar en el anonimato y al lanzarse al margen de unas páginas en permanente metamorfosis, que su labor es circunstancial, construye, al mismo tiempo, el valor, el significado del autor al que comenta ${ }^{24}$.

Pareciera ser que es esa falta de autoría-autoridad la que da fundamento a la actividad de G3. El glosador no es responsable de lo que escribe, pero no sólo porque se deslinda de su valor de verdad, sino porque se configura a sí mismo como un vehículo para hacer del margen un espacio intertextual. No es él quien escribe «aquí», ni quien da datos contradictorios, sino otros, otros textos, otros autores, que aparecen en el espacio marginal sin que quiera plasmarse, mucho menos identificarse, una voluntad que pone a esos textos en diálogo. Esto se vuelve más radical si se recuerdan los elementos de ficcionalización presentes en la segunda glosa: esa adjetivación legendaria no es obra de un autor, sino obra de la tradición. Se ve cómo la tradición dice y debe decir ciertas cosas, de cómo se narra, y así de hecho se convierte también en tradición textual lo que antes llamábamos subjetividad del glosador: no es la subjetividad del glosador, sino una forma tradicional, estipulada de la subjetividad.

G3 está dando cuenta entonces de una instancia de la lectura del códice, que se produce seguramente hacia el 1700, en el que la circulación de la palabra manuscrita parecería revestir algunos matices particulares. Aparece un valor por la literalidad, por la exactitud del testimonio y la precisión de la referencia al original que, si bien dista de ser un valor inapelable, parece tener alguno. Esa precisión de la cita no está debida, al parecer, y eso nos indica la deixis de la primera cita, a una enajenación de la palabra citada, que es el uso al que estamos acostumbrados, sino casi a lo contrario: a la apropiación de un texto otro, pero no por parte del autor, o de la mano, sino de parte de un universo discursivo intertextual en el que los textos se cruzan y se iluminan. Tal intertextualidad no está, entonces, al servicio de fortalecer las credenciales de una verdad histórica, sino al servicio de la leyenda, de aquello que merece ser transmitido por su valor mismo como objeto discursivo. Lo que nos muestra ya, entre otras cosas, que para esta época la Crónica de la población de Ávila ya no debe ser leída como testimonio histórico (quizás tampoco su contemporáneo Fernández Valencia), sino que la historiografía medieval se transforma lentamente en un objeto a ser tratado con las herramientas de la ficción.

\footnotetext{
${ }^{24}$ RODRÍGUEZ VELASCO, Jesús D., «La Bibliotheca y los márgenes: Ensayo teórico sobre la glosa en el ámbito cortesano del siglo XV en Castilla. I: códice, dialéctica y autoridad », in eHumanista, $\mathrm{n}^{\circ}$ 1, 2001, p. 119-134, cita en p. 129.
} 